\title{
CAVO-CAVOSTOMIA TERMINOLATERAL INFRA-HEPÁTICA EM RECEPTORES DE TRANSPLANTE DE FÍGADO
}

\author{
Infrahepatic Terminolateral Cavocavostomy in liver transplant recipients \\ Olival Cirilo Lucena da Fonseca Neto, Renatha Inácia Parente, Priscylla Rabelo, Paulo Sérgio Vieira de Melo, \\ Américo Gusmão Amorim, Cláudio Moura Lacerda.
}

\begin{abstract}
RESUMO
Introdução: O transplante de fígado é o único tratamento definitivo para as doenças hepáticas terminais. A cavocavostomia terminolateral infra-hepática é uma técnica proposta para o transplante hepático e derivada do piggy back clássico, utilizada como técnica única ou como recurso em casos de obstrução, estenose ou anatomia desfavorável das veias hepáticas, possibilitando dessa maneira uma alternativa para o implante da veia cava inferior. Objetivo: Descrever a experiência de um centro especializado utilizando a cavo-cavostomia terminolateral infra-hepática, principalmente em termos de resultados e complicações em intraoperatório e pós-operatório. Métodos: Estudo retrospectivo de pacientes adultos submetidos ao transplante de fígado, através da técnica cavo-cavostomia terminolateral infra-hepática, no período de 1999 a 2018. Resultado: Foram encontrados 10 pacientes submetidos à técnica, de 28 a 70 anos, classificados em eutrófico e sobrepeso e MELD de 10 a 47. Em sete pacientes, a cavo-cavostomia terminolateral infra-hepática foi a técnica única de escolha e os demais foram associados a outra técnica. Não foi encontrado em nenhum dos pacientes dificuldade no desenvolvimento da cavo-cavostomia terminolateral infra-hepática, ou complicações em intra e pós-operatório decorrentes desta. Conclusão: A técnica utilizada neste trabalho é uma alternativa a ser considerada para reconstrução caval, em situações como indisponibilidade anatômica e complicações do fluxo de saída das veias hepáticas.
\end{abstract}

Descritores: Transplante de Fígado; Cirurgia; Veias Cavas.

Instituição:

Serviço de Cirurgia Geral e Transplante Hepático do Hospital Universitário Oswaldo Cruz - HUOC, Recife, PE, Brasil.

\section{Correspondência:}

Olival Cirilo Lucena da Fonseca Neto

Rua Jacobina , 45, 1002 - CEP- 52011180, Recife/PE.

Tel.: (81) 99989-0208

E-mail: olivalneto@globo.com

Recebido em: 01/10/2018

Aceito em: 31/10/2018

\section{INTRODUÇÃO}

O transplante de fígado ( $T x F)$ é o único tratamento definitivo para as doenças hepáticas terminais. No transplante hepático descrito originalmente por Starzl et al,1 foi utilizada a técnica convencional (CV), em que a veia cava inferior $(\mathrm{VCl})$ retro hepática é removida na hepatectomia do fígado nativo e ocorre a inserção da $\mathrm{VCl}$ do doador. ${ }^{1}$ Desde então, outras técnicas para reconstrução da $\mathrm{VCl}$ têm sido propostas, como o piggyback $(\mathrm{PB})$ e algumas derivações deste..$^{2-7}$

$\mathrm{O}$ PB apresenta algumas vantagens frente à técnica CV, como o fato de evitar a dissecção retrocaval e colaborar para a estabilidade hemodinâmica. ${ }^{8}$ Para a realização da técnica PB clássico, a VCI retro-hepática do receptor deve ser preservada, e a anastomose da VCl supra- 
hepática do enxerto será feita através dos orifícios das veias hepáticas nativas. ${ }^{2-4}$

A cavo-cavostomia terminolateral infra-hepática (CTLI) é uma técnica proposta para o transplante de fígado $(\mathrm{TxF})$ e derivada do PB clássico. A VCl retrohepática é preservada, assim como no PB clássico, e utilizase o orifício infrahepático da $\mathrm{VCl}$ do aloenxerto para a anastomose em um orifício recém criado na parede anterior da $\mathrm{VCl}$ infrahepática do receptor. Esta técnica tem sido utilizada como um recurso em casos de obstrução, estenose ou anatomia desfavorável das veias hepáticas, possibilitando, dessa maneira, uma alternativa para o implante da $\mathrm{VCl}^{7,9,10}$

Neste trabalho, será descrita a experiência de um centro especializado utilizando a CTLI, principalmente em termos de resultados e complicações em intraoperatório e pós-operatório.

\section{MÉTODOS}

Estudo retrospectivo de pacientes adultos submetidos ao transplante de fígado através da técnica CTLI realizado pela Unidade de Transplante de Fígado (UTF) em RecifePE, no período de agosto 1999 a maio de 2018. Pacientes pediátricos (< ou $=12$ anos) submetidos ao transplante de fígado foram excluídas. A imunossupressão padrão adotada para os pacientes consistiu em esquema tríplice de imunossupressão (tracolimus, prednisona e micofenolato de mofetila).
Os dados foram colhidos através de prontuários médicos e os resultados observados durante a evolução clínica.

\section{RESULTADOS}

Os dados demográficos dos pacientes no momento do TxF são apresentados na tabela 1 e as características perioperatórias dos receptores de TxF na tabela 2. Dos 1400 transplantes, foram encontrados 10 pacientes submetidos à técnica CTLI, oito homens e duas mulheres, com faixa etária de 28 a 70 anos, classificados em eutrófico e sobrepeso de acordo com o IMC (índice de massa corporal) ${ }^{11}$ e MELD (Model for End Stage Liver Disease) que variou de 10 a 47.

O diagnóstico predominante de indicação ao transplante foi cirrose por vírus $\mathrm{C}$, vírus $\mathrm{B}$, álcool, NASH (Nonalcoholic Steatohepatitis) e cirrose biliar primária e secundária (lesão iatrogênica de via biliar). Também observou-se hepatocarcinoma, metástase hepática de tumor neuroendócrino, esquistossomose hepatoesplênica, hepatite fulminante e dois retransplantes (não funcionamento primário do enxerto e trombose de artéria hepática). Além disso, os pacientes apresentavam diversas comorbidades pré-operatórias associadas: diabetes mellitus, hipertensão arterial sistêmica, insuficiência cardíaca congestiva, lesão renal aguda, vírus da imunodeficiência humana (HIV) e gestação única tópica pré-termo.

Tabela 1. Dados demográficos dos pacientes no momento do TxF

\begin{tabular}{|c|c|c|c|c|c|c|}
\hline Pacientes & Gênero & Idade (anos) & IMC & Meld & Diagnóstico & Comorbidades \\
\hline$(\mathrm{kg} / \mathrm{m} 2)$ & Meld & $\begin{array}{l}\text { Diagnós- } \\
\text { tico }\end{array}$ & $\begin{array}{l}\text { Comorbi- } \\
\text { dades }\end{array}$ & 15 & Cirrose por vírus $\mathrm{C}+\mathrm{CHC}$ & HAS +DM \\
\hline $\mathrm{P} 2$ & M & 54 & 29,4 & 10 & Cirrose por álcool & DM \\
\hline P3 & $\mathrm{F}$ & 70 & 26,1 & 24 & Cirrose por NASH +EHE & HAS \\
\hline P4 & M & 46 & 27,1 & 25 & Cirrose biliar secundária & Não \\
\hline P5 & M & 54 & 23,6 & 15 & Cirrose por vírus $\mathrm{B}+\mathrm{CHC}$ & Não \\
\hline P6 & M & 68 & 22,8 & 12 & Metástase Hepática de tumor neuroendócrino & ICC \\
\hline P7 & M & 43 & 22,0 & 28 & NFP & HAS + LRA \\
\hline P8 & $\mathrm{F}$ & 28 & 23,8 & 29 & Hepatite fulminante & HIV + GUTPT \\
\hline P9 & M & 41 & 24,4 & 47 & Trombose de Artéria Hepática & Não \\
\hline P10 & M & 64 & 24,1 & 22 & Cirrose por álcool & Não \\
\hline
\end{tabular}

M, masculino; F, feminino;

HAS, hipertensão arterial sistêmica; DM, diabetes mellitus;

NFP, não funcionamento primária do enxerto; EHE, esquistossomose;

ICC, insuficiência cardiaca congestiva; LRA, lesão renal aguda;

HIV, vírus da imunodeficiência humana; NASH, nonalcoholic steatohepatitis;

GUTPT, gestação única tópica pré termo; $\mathrm{CHC}$, hepatocarcinoma. 
Sete pacientes foram submetidos unicamente à técnica CTLI para reconstrução caval, dois pacientes foram submetidos a CTLI associada à cavo-cavostomia terminolateral supra-hepática (CTLS), denominada neste trabalho como CTLI de resgate e, em dois casos, foi realizada a CTLI em retransplante. Não foi encontrado em nenhum dos pacientes dificuldade no desenvolvimento da CTLI, ou complicações em intra e pós-operatório decorrentes desta técnica.

As reconstruções da veia porta e artéria hepática foram finalizadas através de anastomose termino-terminal. Em um paciente foi necessária realização de conduto arterial. A revascularização hepática foi feita de maneira retrógrada em quatro destes; nos demais, procedeu-se revascularização portal. Observou-se síndrome pós- reperfusão em três pacientes. O tempo de cirurgia variou entre 174 a 350 minutos e a fase anepática, entre 20 a 72 minutos. Observou-se produção de bile intraoperatória, em oito pacientes.

Do total dos 10 pacientes, quatro evoluíram para óbito. O não funcionamento primário do enxerto (NFP) e sepse de origem indefinida foram as causas da evolução desfavorável.

Essa análise também levou em consideração o número de pacientes cadastrados no REREME que apresentavam sorologia para citomegalovírus (CMV). Na Tabela 3, observa-se que $40 / 82(48,78 \%)$ apresentavam sorologia positiva para CMV, dos quais, 24/40 (60\%) eram indivíduos do gênero masculino, distribuído em várias faixas etárias.

Tabela 2 - Características Peri operatórias dos receptores de TxF

\begin{tabular}{|c|c|c|c|c|c|c|c|c|}
\hline Pacientes & $\begin{array}{l}\text { Técnica } \\
\text { cirúrgica }\end{array}$ & $\begin{array}{l}\text { Anastomose } \\
\text { biliar }\end{array}$ & $\begin{array}{l}\text { Produziu } \\
\text { bile }\end{array}$ & $\begin{array}{l}\text { Perfusão } \\
\text { hipotérmica } \\
\text { com soro } \\
\text { fisiológico }\end{array}$ & Reperfusão & $\begin{array}{c}\text { Síndrome } \\
\text { pós } \\
\text { reperfusão }\end{array}$ & $\begin{array}{c}\text { Tempo de } \\
\text { cirurgia } \\
\text { (minutos) }\end{array}$ & $\begin{array}{c}\text { Fase } \\
\text { anepática } \\
\text { (minutos) }\end{array}$ \\
\hline $\mathrm{P} 1$ & CTLI & $\mathrm{C}-\mathrm{C}$ & Sim & Sim & Retrógrada & Não & 320 & 70 \\
\hline P2 & CTLI & $\mathrm{C}-\mathrm{C}$ & Sim & Não & Portal & Não & 295 & 41 \\
\hline P3 & CTLI de resgate & $\mathrm{C}-\mathrm{C}$ & Sim & Não & Retrógrada & Não & 310 & 52 \\
\hline P4 & CTLI & $C-D$ & Sim & Não & Retrógrada & Não & 350 & 42 \\
\hline P5 & CTLI & $\mathrm{C}-\mathrm{C}$ & Não & Não & Portal & Sim & 285 & 39 \\
\hline P6 & CTLI & $\mathrm{C}-\mathrm{C}$ & Sim & Não & Portal & Não & 340 & 50 \\
\hline $\mathrm{P} 7$ & CTLI em ReTx & $\mathrm{C}-\mathrm{C}$ & Sim & Sim & Portal & Sim & 174 & 20 \\
\hline P8 & CTLI & $\mathrm{C}-\mathrm{C}$ & Sim & Não & Retrógrada & Não & 305 & 30 \\
\hline P9 & $\begin{array}{c}\text { CTLI de resgate } \\
\text { em } \operatorname{ReTx}\end{array}$ & C-D & Não & Sim & Retrógrada & $\operatorname{Sim}$ & 300 & 72 \\
\hline P10 & CTLI & $\mathrm{C}-\mathrm{C}$ & Sim & Não & Retrógrada & Não & 345 & 20 \\
\hline
\end{tabular}

CTLI, cavostomia terminolateral infra-hepática; ReTx, retransplante; C-C, colédoco-colédoco; C-D, colédoco-duodeno;

\section{DISCUSSÃO}

Não há, hoje, um consenso sobre a técnica ideal de reconstrução da $\mathrm{VCl}^{12}$ Alguns autores defendem que a PB ofereça vantagens sobre a técnica $C V$, já que a $\mathrm{CV}$ requer o clampeamento completo da $\mathrm{VCl}$ infra-diafragmática e da veia porta, com possíveis consequências hemodinâmicas resultantes da congestão de todo território inferior do corpo, além da necessidade de dissecção retrocaval. ${ }^{13,8}$ Porém, também há complicações passíveis de ocorrer, durante o PB, como obstrução de fluxo das veias hepáticas. ${ }^{14}$

A CTLI é uma alternativa em casos de complicações do fluxo de saída das veias hepáticas, após um PB ou
CTLS, a fim de alcançar vazão suficiente ou é também realizada como técnica única de escolha, através do fechamento do orifício superior da $\mathrm{VCl}$ do enxerto, ${ }^{6,7,10}$ ambos descritos neste trabalho (Figura 1).

Foi desenvolvido com segurança o uso da CTLI em ambos os pacientes descritos, assim como em trabalhos anteriores, $, 7,10$ com complicações não relacionadas à técnica, que resultou em NFP.

O presente trabalho descreve também o caso de uma paciente com GUTPT, e submetida a TxF através da CTLI, desconhecendo-se na literatura, até então, o uso da CTLI em caso semelhante. 
Figura 1 - Comparação entre técnica convencional (CV), cavostomia terminolateral supra hepática (CTLS), cavostomia terminolateral infra-hepática CTLI e cavostomia terminolateral infra-hepática de resgate (CTLI de resgate).

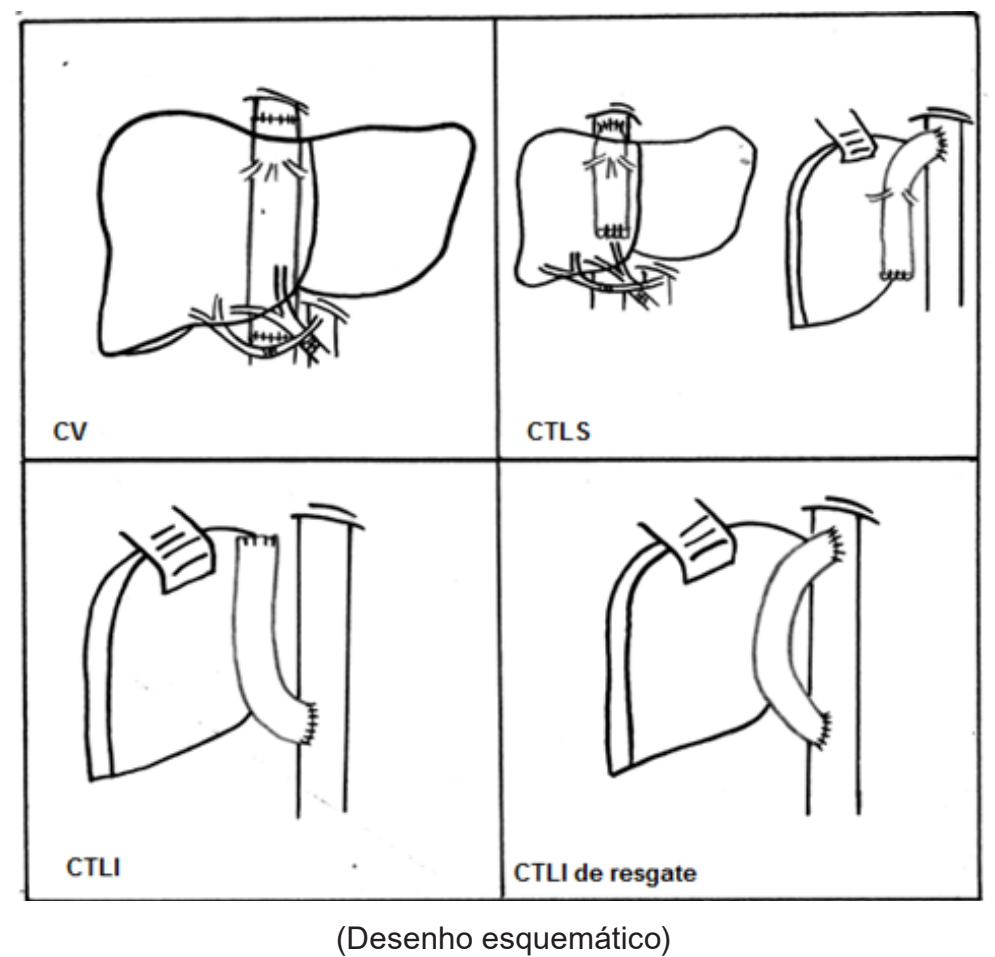

\section{CONCLUSÃO}

A técnica para reconstrução caval CTLI é uma alternativa a ser considerada em casos de complicações do fluxo de saída das veias hepáticas ou indisponibilidade anatômica, não sendo encontrado neste trabalho complicações específicas relacionadas a técnica.

\section{ABSTRACT}

Introduction: Liver transplantation is the only definitive treatment for terminal liver disease. Intrahepatic terminolateral cavostomy is a proposed technique for liver transplantation and derived from the classic piggyback, used as a single technique or as a resource in cases of obstruction, stenosis or unfavorable anatomy of the hepatic veins, thus providing an alternative for the implantation of the inferior vena cava. Purpose: To describe the experience of a specialized center using infrahepatic terminolateral cavostomy, mainly in terms of intraoperative and postoperative outcomes and complications. Methods: Retrospective study of adult patients who underwent liver transplantation through the infrahepatic terminolateral cavostomy technique from 1999 to 2018 . Results: We found 10 patients undergoing the technique, aged 28 to 70 years, classified as eutrophic and overweight and MELD from 10 to 47. In seven patients, infrahepatic terminolateral casvostomy was the only technique chosen and in the others it was associated with another technique. It was not found in any of the patients difficulty in developing the infrahepatic terminolateral cavostomy or intra and postoperative complications resulting from it. Conclusion: The technique used in this study is an alternative to be considered for cava reconstruction, in situations such as anatomical unavailability and complications of outflow from the hepatic veins.

Keywords: Liver Transplantation; Surgery; Venae Cavae. 
Olival Cirilo Lucena da Fonseca Neto, Renatha Inácia Parente, Priscylla Rabelo, Paulo Sérgio Vieira de Melo, Américo Gusmão Amorim, Cláudio Moura Lacerda.

\section{REFERÊNCIAS}

1. Starzl TE, Marchioro TL, Vonkaulla KN, Hermann G, Brittain RS, Waddell WR. Homotransplantation of the liver in humans. Surg Gynecol Obstet. 1963;117:659-76.

2. Calne RY, William R. Liver transplantation in man. I. Observation on technique and organization in five cases. $\mathrm{Br}$ Med J. 1968;4:535-40.

3. Starzl TE, Putnam CW. Experience in Hepatic Transplantation. Philadelphia, PA: WB Saunders Company.1969;131.

4. Tzakis A, Todo S, Starzl TE. Orthotopic liver transplantation with preservation of the inferior vena cava. Ann Surg.1989;210:649-52.

5. Belghiti J, Panis Y, Sauvanet A, Gayet B, Fékété F. A new technique of side to side caval anastomosis during orthotopic hepatic transplantation without inferior vena caval occlusion. Surg Gynecol Obstet. 1992;175:270-2.

6. Cherqui D, Lauzet JY, Rotman N, Duvoux C, Dhumeaux $D$, Julien $M$, et al. Orthotopic liver transplantation with preservation of the caval and portal flows. Technique and results in 62 cases. Transplantation. 1994;58:793-6.

7. Merenda R, Gerunda GE, Neri D, Barbazza F, Marzio ED, Bruttocao A, et al. Infrahepatic Terminolateral CavoCavostomy as a Rescue Technique in Complicated "Modified" Piggyback Liver Transplantation. Elsevier Science Inc. 1997;576-9.

8. Busque S, Esquivel C, Concepcion W, So SK: Experience with the piggyback technique without caval occlusion in adult orthotopic liver transplantation. Transplantation. 1998;65:7782.
9. Lima CX, Garcia SM, Lima MB. Infrahepatic Terminolateral Cavocavostomy: A Case Report. Transplantation Proceedings. 2009;41: 1997-9

10. Kinaci E, Kayaalp C, Yilmaz S, Otan E. Salvage with a Secondary Infrahepatic Cavocavostomy of the Occluded Modified Piggyback Anastomosis during Split Liver Transplantation: A Case Report. Hindawi Publishing Corporation Case Reports in Transplantation. 2014.

11. World Health Organization. Obesity: preventing and managing the global epidemic. Report of a World Health Organization Consultation. Geneva: World Health Organization. 2000;1253.

12. Gurusamy KS, Pamecha V, Davidson BR. Piggy-back graft for liver transplantation. Cochrane Database of Systematic Reviews. 2011.

13. Pappas G, Palmer WM, Martineau GL, Penn I, Halgrimson CG, Groth CG et al. Hemodynamic alterations caused during orthotopic liver transplantation in humans. Surgery.1971;70:872-5.

14. Ye Q, Zeng C, Wang Y, Fang Z, Hu X, Xiong Y, et al. Risk Factors for Hepatic Venous Outflow Obstruction in Piggyback Liver Transplantation: The Role of Recipient's Pattern of Hepatic Veins Drainage into the Inferior Vena Cava. Annals Of Transplantation. 2017;22:303-8. 